\title{
The Absence of Universally-Conserved Protein-coding Genes
}

Change Laura Tan

Division of Biological Sciences 102 LeFevre Hall

University of Missouri

Columbia, MO 65211

USA

Phone: 573-882-1581

Email: tanc@missouri.edu 


\section{Abstract}

2 Public access to thousands of completely sequenced and annotated genomes provides a great

3 opportunity to address the relationships of different organisms, at the molecular level and on a

4 genome-wide scale. Via comparing the phylogenetic profiles of all protein-coding genes in 317

5 model species described in the Ortholnspector3.0 database, we found that approximately

$629.8 \%$ of the total protein-coding genes were orphan genes (genes unique to a specific species)

7 while $<0.01 \%$ were universal genes (genes with homologs in each of the 317 species

8 analyzed). When weighted by potential birth event, the orphan genes comprised $82 \%$ of the

9 total, while the universal genes accounted for less than $0.00008 \%$. Strikingly, as the analyzed

10 genomes increased, the sum total of universal and nearly-universal genes plateaued while that

11 of orphan and nearly-orphan genes grew continuously. When the compared species increased

12 to the inclusion of 3863 bacteria, 711 eukaryotes, and 179 archaea, not one of the universal

13 genes remained. The results speak to a previously unappreciated degree of genetic biodiversity,

14 which we propose to quantify using the birth-event-weighted gene count method.

15 Keywords: conserved genes, universal genes, orphan genes, taxonomically-restricted 


\section{Introduction}

The rapid advances of whole genome sequencing technologies have facilitated comparative and evolutionary analyses, at fine molecular detail, mostly based on comparisons of similarities of DNA, RNA, or protein sequences, e.g., homologous genes or gene contents.

Many methods have been developed to identify homologous genes. By definition, any gene and its homologs found in other species are derived from a common ancestral gene. Since the history of genes are generally not known, homology-identification is challenging. Consequently, different methods that are based on different assumptions and algorithms may differ in

27 homology classifications. Ortholnspector is one of the three most balanced methods (the other two are InParanoid and Hieranoid) of orthology inference in specificity and sensitivity (1-3).

Ortholnspector identifies orthologs by dividing genes into inparalog groups based on all-to-all proteome BLAST comparisons and then searching for a reciprocal-best-hit relationship between inparalog groups (1). Since it does not require a reciprocal-best-hit between individual genes it is more sensitive than many other methods, including Inparanoid and OrthoMCL $(1,2,4,5)$. organisms (3863 bacteria, 711 eukaryotes, and 179 archaea), generating an orthology resource with the broadest species coverage (except viruses) (6). Of the 4753 organisms, 317 (144 eukaryotes, 142 bacteria, and 31 archaea) are deemed model species (referred to as NMS

37 (Nevers's Model Species) hereafter) either due to their importance in the biological field or due to a consideration of taxonomic coverage. Orthologous genes across three domains of life are available in the Ortholnspector website for these species. For a non-model species, only orthologous genes within its own domain of life are available.

41 To investigate the relationship of gene contents of different species, we compared the phylogenetic profiles of all NMS. A phylogenetic profile of a protein describes the presence or 
absence of its homologs across a given set of organisms (7). Two proteins with the same phylogenetic profiles tend to function in the same biological process, though the accuracy of the functional-linkage prediction depends on the criteria of defining homology and selection of reference species (7-11). We focused our attention to proteins with two extreme distribution: universal or orphan. To accommodate the potential bias of the method used to identify homologous genes and the consequence of occasional gene loss or genome sequencing or annotation errors, we have also analyzed nearly-universal genes and nearly-orphan genes.

We discovered an unexpected pattern of the distribution of universal genes and orphan genes. We found that every species has a large number of orphan and nearly-orphan genes, but none, or only a few, universal and nearly-universal genes. Contrary to the common expectation that homologs would be found for orphan genes so that orphan gene number would decrease as more species are analyzed, the number of orphan genes grows continuously; each addition of species brings new orphan genes, though often resulting in a decrease of universal genes. not a single universal gene maintained its status as a universal gene when enough species were sampled. In other words, all genes are taxonomically restricted, though at different levels of restriction.

\section{Materials and Methods}

62 Phylogenetic profiles of all the 317 NMS were provided by Nevers and Lecompte in CSV

63 (delimited by tabs). The phylogenetic profile for each species contains information about the presence (indicated with a 1) or absence (indicated with a 0) of homologs in the 317 NMS (columns) for all its protein-coding genes (rows). For the column that corresponds to the species 
shifted one cell to the left. After that was corrected, the phylogenetic profiles were saved as Excel files. Number of species in which there are orthologous genes for a NMS protein was calculated based on the phylogenetic profiles using Microsoft Excel and/or a script written for this project by Andrew Jones. Identity of proteins and that of their orthologs were manually curated from the Ortholnspector website (https://lbgi.fr/orthoinspectorv3/). Gene function annotations were mostly from the UniProt Knowledgebase (UniProtKB, https://www.uniprot.org/uniprot/), occasionally from organism-specific databases, e.g., the Drosophila Genome Database (https://flybase.org/) and the Saccharomyces Genome Database (https://www.yeastgenome.org/). All figures were generated using Microsoft Excel and PowerPoint.

\section{Categorizing Genes according to the Numbers of Species Having Their Homologs}

The number of species having homologs for a specific gene is the sum total for the row to which that gene belongs, since the presence or absence of its homologs in a species (except its own host or home species) is indicated with 1 or 0 , respectively. Note that the presence of multiple homologs in a species does not increase the count beyond 1. A gene was called an orphan, orphan +1 , orphan $+2 \ldots$ or a universal $(0+1,0+2 \ldots$ universal, "o" means orphan) gene if the sum total is $0,1,2 \ldots$ or 316 .

\section{Identification of Orphan, Nearly-orphan, Universal, and Nearly-universal Genes}

If the number of species having homologs for a specific gene is 316 , then that gene is a universal gene, since it has homolog(s) in every organism analyzed. If the number is 0 , then that gene is an orphan gene, a gene unique to a species; no homolog exists in any of the other species analyzed. Nearly-universal genes are genes conserved in all but five or fewer species analyzed, i.e., a sum total of $315,314,313,312$, or 311 (corresponding to u-1, u-2, u-3, u-4, u-5 genes, "u" means universal). Nearly-orphan genes, by contrast, are genes that are shared by no 
91 more than five of the species analyzed, i.e., a sum total of 1, 2, 3, 4, or 5 (corresponding to o+1,

$92 \mathrm{o}+2, \mathrm{o}+3, \mathrm{o}+4, \mathrm{o}+5$ genes).

\section{Weighted Counts of Genes by Potential Birth Event}

94 The weighted value of a gene is the inverse of the number of species, including the gene's

95 home species, that have homologs for that gene. Thus, an orphan gene was counted as one

$96(=1 / 1)$ gene, while a universal gene was counted as $0.003155(=1 / 317)$ gene. An o+x gene was

97 counted as $1 /(1+x)$ gene, $x$ is any integer between 1 and 316 .

\section{$99 \quad$ Results}

100

101

102

103

104

105

106

107

108

109

\section{Distribution of Total Genes}

To gain a broad view of the species being analyzed, we compared the sizes of their proteomes

(Fig 1). Not surprisingly, on average, eukaryotes have much larger proteome sizes than bacteria and archaea. Eukaryote proteome sizes also vary the most.

Fig 1. Number of protein-coding genes of NMS. Each data point represents a species. Species are arranged according to the order of (6). The first two numbers underneath the name of each domain of life are the number of the model species and that of the total species analyzed in the corresponding domain.

\section{Grouping Genes according to the Occurrences of Their Homologs}

Next, we placed every protein-coding gene encoded in the genomes of all NMS into orphan, orphan +1 , orphan $+2 \ldots$ or universal groups $(0,0+1,0+2 \ldots$ universal) based on whether the number of species in which its homologs exist is $0,1,2 \ldots$ or 316 (S2 Table). Surprisingly, for every species analyzed, the group with most members was the orphan group, and the number 
114 of genes in a group quickly dropped into a handful or even zero with the increase of species

115 containing the corresponding homologs (Fig 2 and S1 Fig).

Fig 2. The number of genes in a group decreases rapidly as the number of species sharing the

117 corresponding homologs increases. Note that the first vertical line in each panel is not its Y-axis but

118 the orphan gene number in the corresponding organism. The numbers in the parenthesis next to the

119 name of a species in each panel are the number of its orphan genes, all protein-coding genes, and the

120 percent of orphan genes. Only five species are shown here. More examples can be found in S1 Fig and

121 S2 Table.

122

\section{Distribution of Universal, Nearly-universal, Orphan, and Nearly-orphan Genes}

124 In order to comprehend the above data, we focused our attention on orphan, nearly-orphan, 125 universal, and nearly-universal genes. A nearly-universal gene is conserved in all but five or 126 fewer species analyzed, while a nearly-orphan gene is shared by no more than five of the 127 species analyzed. As one would expect from a quick glance at Fig 2, S1 Fig, and S2 Table, the 128 number of orphan and nearly-orphan (ONO) genes greatly exceeded that of universal and 129 nearly-universal (UNU) genes. Table 1 lists the ONO, UNU, and the proteome of our seventeen 130 chosen organisms, including three bacteria, three archaea, three plants, one fungus, and seven

131 animals. S3 Table lists the ONO, UNU, and the proteome of all NMS. Fig 3 shows the

132 percentages of ONO genes (colored sections at the bottom), UNU genes (colored sections at

133 the top), and all other genes (gray) in each of the model species. Note the great portion of the

134 ONO genes. In contrast, the portion of the UNU genes are barely visible, especially for 135 eukaryotes. 
Table 1: Numbers of orphan, nearly-orphan, universal, and nearly-universal genes in seventeen chosen organisms

\begin{tabular}{|c|c|c|c|c|c|c|c|c|c|c|c|c|c|}
\hline Species & 0 & $0+1$ & $0+2$ & $0+3$ & $0+4$ & $0+5$ & $u-5$ & $u-4$ & $u-3$ & $u-2$ & $u-1$ & $u$ & Proteome \\
\hline Escherichia coli & 332 & 255 & 177 & 97 & 85 & 95 & 4 & 3 & 4 & 2 & 3 & 1 & 4306 \\
\hline Caulobacter crescentus & 635 & 130 & 99 & 86 & 81 & 74 & 5 & 1 & 2 & 0 & 6 & 0 & 3715 \\
\hline Mycoplasma genitalium & 114 & 16 & 11 & 4 & 5 & 3 & 5 & 2 & 2 & 4 & 5 & 2 & 483 \\
\hline Sulfolobus solfataricus & 843 & 169 & 108 & 102 & 69 & 51 & 3 & 3 & 2 & 2 & 4 & 1 & 2924 \\
\hline $\begin{array}{l}\text { Methanocaldococcus } \\
\text { jannaschii }\end{array}$ & 353 & 94 & 60 & 59 & 36 & 34 & 4 & 4 & 4 & 5 & 3 & 4 & 1787 \\
\hline Haloferax volcanii & 700 & 311 & 265 & 248 & 116 & 60 & 5 & 4 & 3 & 2 & 4 & 4 & 3982 \\
\hline Zea mays & 10719 & 1869 & 1933 & 1033 & 863 & 761 & 3 & 0 & 0 & 0 & 0 & 0 & 39154 \\
\hline Oryza sativa & 19132 & 2380 & 1847 & 958 & 740 & 627 & 2 & 2 & 0 & 0 & 0 & 0 & 43550 \\
\hline Arabidopsis thaliana & 5057 & 893 & 558 & 544 & 546 & 545 & 0 & 1 & 0 & 0 & 0 & 0 & 27252 \\
\hline $\begin{array}{l}\text { Saccharomyces } \\
\text { cerevisiae }\end{array}$ & 1955 & 225 & 128 & 61 & 45 & 44 & 3 & 2 & 0 & 0 & 1 & 0 & 6721 \\
\hline Caenorhabditis elegans & 9418 & 1285 & 994 & 355 & 201 & 180 & 2 & 1 & 0 & 0 & 1 & 0 & 20071 \\
\hline $\begin{array}{l}\text { Drosophila } \\
\text { melanogaster }\end{array}$ & 3189 & 525 & 269 & 250 & 238 & 195 & 1 & 4 & 0 & 0 & 0 & 0 & 13704 \\
\hline Danio rerio & 2808 & 744 & 496 & 488 & 449 & 339 & 0 & 2 & 0 & 0 & 0 & 0 & 24929 \\
\hline Canis lupus & 973 & 171 & 188 & 283 & 311 & 534 & 2 & 1 & 0 & 0 & 0 & 0 & 19756 \\
\hline Mus musculus & 1414 & 972 & 489 & 488 & 510 & 658 & 2 & 2 & 0 & 0 & 0 & 0 & 22274 \\
\hline Homo sapiens & 896 & 719 & 288 & 325 & 402 & 548 & 1 & 2 & 0 & 0 & 0 & 0 & 21006 \\
\hline Gallus gallus & 1136 & 143 & 150 & 79 & 87 & 77 & 1 & 2 & 0 & 1 & 1 & 0 & 15913 \\
\hline
\end{tabular}

Fig 3. Distribution of ONO and UNU genes of all NMS. Each column represents a species whose order

141 of appearance is according to that of (6). Not all organisms are labeled and the species names are too

142 small to read in this figure due to the limitation of space. For a higher resolution figure with all organisms

143 legibly labeled, see the big graph located at the bottom of S3 Table.

145 Fig 4 is a representation of the grouping of genes of all NMS together. Amazingly, the ONO genes represent $42.7 \%(1,228,529)$ of the total $(2,874,537)$, with the orphan group itself occupies about $29.8 \%(855,723)$ of the total. The UNU groups account for less than $0.14 \%$ $(3,906)$ of the total, with the universal group about $0.01 \%(261)$ of the total. whole. 
151 Strangely, the number of universal genes, 261 , is even smaller than the number of model

152 species, 317 . This creates a contradiction with the definition of universal genes, since, by

153 definition, if one true universal gene existed in the model species, then we should have 317

154 universal genes, because a universal gene should (again, by definition) possess a homolog in

155 each of the other model species. To make the situation worse, more than half of the model

156 species have none, while some of them have several universal genes (Table 2). For example,

157 Candidatus caldiarchaeum has seven universal genes, almost twice as many as the ten species

158 with the second largest number (four) of universal genes. Although eukaryotic proteomes are 159 generally much larger than bacterial and archaeal proteomes, they have the least number of 160 universal genes. Of the 144 model eukaryotes, only ten have universal genes and none has 161 more than one universal genes.

Table 2: Distribution of species with zero to seven universal genes

\begin{tabular}{|c|r|r|r|r|r|}
\hline Domain of Life & Bacteria & Archaea & Eukaryotes & All \\
\hline $\begin{array}{c}\text { Number of Model Species } \\
\text { Number of Universal } \\
\text { Genes }\end{array}$ & 0 & 142 & 31 & 144 & 317 \\
\hline & 1 & 59 & 1 & 134 & 168 \\
\hline & 2 & 45 & 4 & 10 & 73 \\
\hline & 3 & 3 & 9 & 0 & 54 \\
\hline 4 & 2 & 8 & 0 & 11 \\
\hline & 5 & 0 & 0 & 0 & 10 \\
\hline & 6 & 0 & 0 & 0 & 0 \\
\hline & 7 & 0 & 1 & 0 & 0 \\
\hline Universal Genes per Genome & 1.17 & 2.74 & 0.07 & 0.82 \\
\hline
\end{tabular}

\section{Weighted Distribution of UNU and ONO Genes} words, they shared one "birth event". Therefore, it is only logical that genes should not have been counted equally; they should be weighted according to their birth events. Consequently, each orphan gene shares its birth right with no other sequence, and should be counted once, while each $0+1$ gene shares its birth right with an ortholog, and should be counted as 0.5 , and 
so on. When thus weighted, the ONO genes represent about $95 \%$ of the total, while the UNU

171 groups represent only about $0.0012 \%$ (Fig 5 and S4 Table). The orphan group itself makes up

172 more than $82 \%$ of the total. In comparison, without weighting, when every gene is counted

173 equally in each species, the ONO genes represent about $43 \%$ of the total, while the UNU genes

174 account for about $0.14 \%$.

175 Fig 5. Distribution of weighted orphan, nearly-orphan, universal, and nearly-universal genes of $176 \quad$ NMS as a whole.

179 To determine the accumulated number of UNU, ONO, and all the protein-coding genes as more 180 species were added, we simply summed the UNU, the ONO, and the proteome of each of the 181 model species, one-by-one, in the sequence of the Nevers's original species order. The number 182 of UNU grew quickly initially with the addition of species, but the growth slowed down soon, and 183 almost plateaued at around 3,300 genes (Fig 6A). Strikingly, the ONO number increased

184 continuously, at a much greater speed than the initial, fastest, growth rate of the UNU number

185 (Fig 6B, orange). Viewed with the same scale, the number of UNU genes appears to show a 186 trend, or slope, of zero (i.e., unchanging along the vertical axis) (Fig 6B, blue).

187 Fig 6. Change of the number of UNU, ONO, and all the protein-coding genes as new organisms 188 were added. Each data point represents a species whose coordinates are $(X, Y)$ and whose encoded protein number is $\mathrm{Pn} . \mathrm{X}$ is the sum of the proteins encoded by all the organisms up to and including that species. $Y$ is the sum of the ONO or UNU of these organisms. 


\section{Identity of the Universal Genes}

193 The numbers of different groups of genes are interesting and important to know but their identity

194 is even more informative for our understanding of life. Here we will describe the identity of the

195 universal genes, while that of the orphan genes will be described in another publication.

196 We first analyzed the homologs of the universal genes of our chosen 17 . We observed that the

197 vast majority of the homologs of a gene encode proteins perform the same function in different 198 organisms, when functional data are available (S5 Table). This indicates the specificity of

199 homolog inference of Ortholnspector. The occasional out-of-place homologs, e.g., the

200 Escherichia coli lysine-tRNA ligase amongst the homologs of asparagine-tRNA ligase and the

201 Caulobacter crescentus peptide chain release factor 3 in the mist of elongation factor $\mathrm{G}$

202 homologs, indicates the ability of Ortholnspector to recognize even very low similarity. For

203 example, the E. coli lysine-tRNA ligase, though not an asparagine-tRNA ligase, belongs to the

204

205

206

207

208

209

210

211

212 same subgroup (class Ilb) of tRNA ligases as the asparagine-tRNA ligase, with the sole other member of this group being aspartate-tRNA ligase (12). These three tRNA ligases recognize related anticodons: aspartate GUC, asparagine GUU, and lysine UUU triplets. The C. crescentus peptide chain release factor 3 belongs to the same subfamily of GTPases and probably has similar structure and binds to overlapping regions of the bacterial ribosomes as the elongation factor $\mathrm{G}(13-15)$. The fact that most homologs of a gene encode proteins with the same characteristics and/or functions demonstrates that Ortholnspector is a reliable method to identify homologs.

The second phenomenon we observed is that the homolog of a universal gene is commonly not a universal gene. This is surprising. However, it explains why the number of universal genes is not an integer multiplication of the number of model species (317), though not why it is smaller than 317. 
216 The third observation is that none of the universal genes in our chosen 17 kept their status as

217 universal genes when checked against their in-domain non-model species in Ortholnspector (S5

218 Table).

219 Next, we expanded our analysis to all the model species. Consistent with the observation of the

220 chosen 17, all universal genes lost their status as a universal gene when checked against their

221 in-domain non-model species in Ortholnspector.

222 The protein characteristics of five universal genes (Q74MY3_NANEQ, R1E424_9ARCH,

223 E4WXB9_OIKDI, C4V6P4_NOSCE, A2ER26_TRIVA) were not clear. To solve this problem, we

224 examined their homologs in the chosen 17 (S6 Table). Assuming a gene's homologs share its

225 identity, we called Q74MY3_NANEQ and R1E424_9ARCH Obg-like ATPase 1, A2ER26_TRIVA

226 and C4V6P4_NOSCE elongation factor 2, and E4WXB9_OIKDI isoleucine-tRNA ligase.

227 With the new assignation for these five, the 261 universal genes encode eight proteins:

228 aspartate-tRNA ligase, phenylalanine-tRNA ligase alpha subunit, valine-tRNA ligase, isoleucine-

229 tRNA ligase, elongation factor G (name according to bacteria, corresponding to the archaeal

230 and eukaryotic elongation factor 2), elongation factor Tu (name according to bacteria,

231 corresponding to the archaeal and eukaryotic elongation factor 1), DNA-directed RNA

232 polymerase subunit beta, and Obg-like ATPase 1 (Table 3 and S7 Table). Five of these eight

233 belong to the Up-to-date bacterial core gene set (the two elongation factors and valine-tRNA

234 ligase do not) (16). 
Table 3: Identity of universal genes and their distribution in the three domains of life

\begin{tabular}{|l|l|l|}
\hline Bacteria & Archaea & Eukaryotes \\
\hline Aspartate-tRNA ligase (1) & Aspartate-tRNA(Asp/Asn) ligase (10) & \\
\hline $\begin{array}{l}\text { DNA-directed RNA } \\
\text { polymerase subunit beta (3) }\end{array}$ & $\begin{array}{l}\text { DNA-directed RNA polymerase } \\
\text { subunit beta (5) }\end{array}$ & $\begin{array}{l}\text { DNA-directed RNA polymerase } \\
\text { subunit beta (4) }\end{array}$ \\
\hline $\begin{array}{l}\text { Elongation factor G } \\
\text { (Elongation factor 2) (7) }\end{array}$ & Elongation factor 2 (31) & $\begin{array}{l}\text { Elongation factor 2 (5) } \\
\text { (A2ER26_TRIVA, C4V6P4_NOSCE) }\end{array}$ \\
\hline $\begin{array}{l}\text { Elongation factor Tu } \\
\text { (Elongation factor 1) (57) }\end{array}$ & Elongation factor 1-alpha (11) & $\begin{array}{l}\text { Isoleucine-tRNA ligase (1) } \\
\text { (E4WXB9_OIKDI) }\end{array}$ \\
\hline $\begin{array}{l}\text { Phenylalanine-tRNA ligase } \\
\text { alpha subunit (97) }\end{array}$ & $\begin{array}{l}\text { Phenylalanine-tRNA ligase alpha } \\
\text { subunit (7) }\end{array}$ & \\
\hline Valine-tRNA ligase (1) & & $\begin{array}{l}\text { Obg-like ATPase 1 (2) } \\
\text { (Q74MY3_NANEQ, R1E424_9ARCH) }\end{array}$ \\
\hline
\end{tabular}

Note: The numbers in parenthesis after the names of genes indicate the numbers of species in which the corresponding genes were recognized as universal genes. Genes recognized as universal genes in species across three domains are shaded in dark gray and those between two domains in gray. The original names for the five universal genes that we assigned new identity have also been included in the table.

244 Therefore, even though by definition, if there is one universal gene in the model species, we should have 317 such genes because it should have a homologous gene in each of the model species. And all of the 317 homologous genes should be the same, at least very similar. But we

247 have only 261 universal genes and they are not all the same, although they all, except one

248 (Obg-like ATPase 1), are involved in either gene transcription (the DNA-directed RNA polymerase subunit beta) or gene translation (the t-RNA ligases and elongation factors). Even 


\section{Discussion}

255 Our comparisons of the genetic profiles of 317 proteomes revealed that ONO genes are a

256 common occurrence, while the UNU genes are very rare. The more organisms are included in

257 the analysis, the more ONO genes are detected and the smaller the percentage of UNU gene

258 becomes. Lastly, not a single universal gene remains its status as universal when enough

259 organisms are included.

\section{Universal vs Non-universal Genes}

261 The continuous increase of the ONO numbers and the leveling off of the UNU numbers is consistent with earlier observations (19-29). These analyses have led to a dramatic shrinking, or even vanishing, of the "universal," or universally-conserved, core set of genes and proteins with a concomitant linear growth in the so-called "orphan" or "taxonomically restricted" sequences. "Accordingly," notes Koonin, "the universal core of life has shrunk almost to the point of vanishing"(30). Indeed, after complete sequencing of the first two bacterial genomes, a comparison of the 1727 protein-coding genes of Haemophilus influenza and the 468 conserved homologous genes decreased to 63 (25). With the inclusion of 1000 genomes, the

271 number of universally-conserved homologous genes became zero - not a single protein-coding 272 gene was conserved across the 1000 prokaryotes compared (26).

273 What is surprising, and counterintuitive, is that the homologs of universal genes normally are not

274 universal genes. This results from how we define and detect homologs. Two genes are deemed

275 homologous as long as part of their encoded proteins share some sequence similarity, normally

276 an e-value of $10^{-3}$ to $10^{-5}$ in a BLASTp search. Ortholnspector uses an e-value cutoff of $1 e^{-9}$

277 (about $\left.1.2 \times 10^{-4}\right)(1)$. At this condition, the C. crescentus peptide chain release factor 3 and 
elongation factor $\mathrm{G}$ are detected as homologs because they share some sequence homology in their GTPase domains. With the normal e-value cutoffs, the well-known Drosophila orphan genes Jingwei and Zeus will not be recognized as orphan genes because their high sequence similarity with other wildly distributed genes (31-38). For example, Drosophila melanogaster Zeus, though without homologs in the bacterial and archaeal model species, has homologs in 100 of Nevers's 143 eukaryotic model species, including Saccharomyces cerevisiae, Caenorhabditis elegans, and Gallus gallus (Tan, unpublished observation).

To illustrate how the homologs of a universal gene can be non-universal genes, opposite to what one would expect by the definition of a universal gene, we made up three hypothetical proteins, $A, B$, and $U$ (Fig 7$)$. $U$ shares parts 1,2 , and 3 with $A$ and parts 1,4 , and 5 with $B$. Each part can be one or a group of amino acids. Under the criterion that two proteins are homologous if they share three parts, $U$ and $A$ are homologs, so are $U$ and $B$. However, $A$ and

B only share part 1 , thus are not homologous. $U$ is a universal gene in this scenario, while $A$ and $B$ are not. How would the ancestor(s) of $A, B$, and $U$ look like, the one on the bottom left, the one on the bottom right, or something else? How can we know?

Fig 7. A possible scenario of how a homolog of a universal gene could be a non-universal gene.

\section{Different Levels of Homology}

The puzzle that homologs of a universal gene are normally non-universal genes necessitates distinguishing different levels of homology. The concept of gene homology and the identification of homologous genes among different species are foundational to our study and numerous other comparative genomic studies. We think that it will be fruitful to define and distinguish different levels of homology. Here we propose the following levels of homology: 1) proteindomains (or partial sequence homology, or regional homology), 2) full length proteins, 3) full length genes, including the 5'- and 3'- untranslated regions and introns, 4) signal transduction pathways, 5) tissues, 6) organs, 7) body parts. The first three levels are at the molecular level 
and concern protein-coding genes. The higher the level of homology two genes share, the more likely they will perform the same function. Each level has its own value, even the lowest level, the level one. For instance, if two proteins both have a kinase domain, then they will be a kinase

307 of some sort. Their other non-homologous regions may determine their substrates and their 308 cellular locations of functioning. If these two proteins share homology throughout their entire 309 length, then we can predict that they possess the same substrate specificity and function in the 310 same cell compartment.

311 Currently, it is a general practice to call two proteins homologous as long as they share level 312 one homology and be put into the same protein family. This can cause unnecessary challenges 313 for functional annotation of genes because thus assigned family members may perform 314 opposite or unrelated functions. This can be confusing, even misleading, especially when thus315 identified "homologous" genes are given the same or similar names. For example, Frizzled, a seven-transmembrane protein, and FrzB (also known as soluble or secreted frizzled-related

317 proteins), a protein that is similar to the amino-terminal cysteine rich domain of Frizzled but has

318 no transmembrane segments, are included in the same protein family (InterPro:

319 https://www.ebi.ac.uk/interpro/entry/InterPro/IPR015526/, PANTHER:

320 http://www.pantherdb.org/panther/family.do?clsAccession=PTHR11309, UniProtKB:

321 https://www.uniprot.org/uniprot/Q92765). However, the former is a Wnt receptor necessary for

322 Wnt signaling, while the latter inhibits Wnt-signaling. A similar example is the C. crescentus

323 peptide chain release factor 3 and the elongation factor $\mathrm{G}$ discussed earlier. They are put into

324 the same subfamily of GTPases but perform different functions. Many bacterial and archaeal

325 genes are called globins because they share partial sequence similarity with hemoglobins, but 326 instead of carrying oxygen around like a hemoglobin, a bacterial globin does not bind oxygen, 327 instead may function as a nitrogen monoxide detoxifier (39). 
We propose to provide the following information when declaring two genes homologous: 1) level of homology, or homology coverage, 2) degree of homology, 3) e-value cutoff, and 4) a visual representation of the homology. Homology coverage should indicate whether the homology level is of protein-domain, full-length protein, or full-length DNA sequence. For example, we may divide every protein into four quarters, 1 to 4 from the $\mathrm{N}$-terminal to the C-terminal. The homology between FrzB and Frizzled can be described as FrzB-p1-4/Frizzled-p1, "p" for protein. Homology degree can be indicated with the percent identity of a BLASTp search. A visual representation of the homology should include both the regions that can be aligned and those that cannot be aligned. Fig 8 depicts a visual representation of homology between two subunits, Rpb A' and A", of archaea Pyrococcus furiosus RNA polymerase and the largest subunit Rpb1 of S. cerevisiae RNA polymerase II. A' aligns with the first two quarters of Rpb1. A" aligns with the third quarter of Rpb1. The C-terminal quarter of Rpb1 is unique to eukaryotes and is essential for eukaryotic gene transcription initiation, elongation, termination and intron splicing (40). Specific information about the levels at which proteins are homologous will not only avoid making incorrect connection of protein functions but also facilitate understanding of true relationship of genes.

Fig 8. A comparison of S. cerevisiae Rpb1 (gray, bottom) with P. Furiosus Rpb A' and A" (green, top). Segment locations are based on amino acid positions of Rpb1 protein. Segments present in Rpb1 but not in Rpb A' and A" are indicated with trapezoids and negative numbers, while those segments

348 The values of the numbers, which correlate with the sizes of the triangles and trapezoids, represent the 349 numbers of amino acids of the corresponding regions that are present in only one of the two proteins compared. The C-terminal tail of Rpb1 that is missing in P. furiosus RNA polymerase is highlighted with a red dash-lined box. The comparison is from Fig 1 l of (40). 


\section{A Novel Index of Gene Diversity}

Our birth-event-weighted gene distribution method can be used as a reasonable indication of the diversity of genes, or different types of genes. Since homologous genes (correctly defined gene number count encoded by all species on earth. The weighting approach corrects this, and thus can be used as an index of the number of gene types or gene diversity. Moreover, weighting is logically required if indeed homologous genes share a common ancestral gene. But this makes the explanation of the origin of ONO genes more acutely mysterious and makes it more important to study their functions, which we will address in the near future.

\section{Limitation of This Study}

364 The number and identity of genes in each of our orphan, $0+1,0+2 \ldots$ and universal homologous groups may be different using a different method and/or a different e-value cutoff for homology calling or when different species are included, though the trend of the differential growth of ONO and UNU genes will not change. It will be interesting to study how the phylogenetic profiles of proteins will change with a change of the criteria of homolog calling, such as the percentage of gene length covered, percentage of identity, alignment gap penalty, e-value cutoff, and calculation models (alignment algorisms) for these parameters. Furthermore, how the phylogenetic profiles would change when different species are included.

372 Recent years have witnessed a growth of interests in orphan genes (19-22, 28, 41-56).

373 However, the increase of interests is incomparable to the increase of orphan gene number.

374 Most of the orphans have unknown functions and will be a rich soil for discovery of new 375 enzymes and/or unknown substrates of known enzymes (57) or new genotype-phenotype 376 connections. The broad existence of orphan genes calls for a greater attention to them from the

377 biological community. 


\section{Conclusions}

379 Our in-depth analysis of phylogenetic profiles of 317 proteomes across the three domains of life

380 shows that ONO genes are a common occurrence in the sense that each organism has a

381 significant number of them, while UNU genes are very rare. Most organisms, especially

382 eukaryotes, do not have any UNU genes. Furthermore, the sum total of UNU genes almost

383 plateaued while the number of ONO genes grew continuously when the number of organisms

384 being analyzed increased. More importantly, every universal gene lost its status as a universal

385 gene when the sampled number of organisms is increased. These results revealed a great

386 challenge to explain not only the origin of genes but also the origin of life and the origin of

387 biodiversity. We propose to use the birth-event-weighted distribution of genes as an indication of

388 gene diversity, even though the weighting makes it more difficult to explain the origin of genes

389 by the common belief that genes were generated via duplication and diversification because of

390 the greatly enlarged portion of the ONO genes.

\section{Acknowledgments}

393 The author thanks Andrew Jones for help with calculating the numbers of homologs, Rob Sadler

394 and Paul Nelson for comments on the manuscript, Alan Marshall and Nicholas Valentine for

395 help with computer. 


\section{References}

1. Linard B, Thompson JD, Poch O, Lecompte O. Ortholnspector: comprehensive orthology analysis and visual exploration. BMC Bioinformatics. 2011;12:11.

402 2. Altenhoff AM, Boeckmann B, Capella-Gutierrez S, Dalquen DA, DeLuca T, Forslund K, et al.

403 Standardized benchmarking in the quest for orthologs. Nat Methods. 2016;13(5):425-30.

4043 3. Liebeskind BJ, McWhite CD, Marcotte EM. Towards Consensus Gene Ages. Genome Biol Evol. 405 2016;8(6):1812-23.

406 4. Li L, Stoeckert CJ, Jr., Roos DS. OrthoMCL: identification of ortholog groups for eukaryotic 407 genomes. Genome Res. 2003;13(9):2178-89.

408 5. O'Brien KP, Remm M, Sonnhammer EL. Inparanoid: a comprehensive database of eukaryotic orthologs. Nucleic Acids Res. 2005;33(Database issue):D476-80.

6. Nevers Y, Kress A, Defosset A, Ripp R, Linard B, Thompson JD, et al. Ortholnspector 3.0: open portal for comparative genomics. Nucleic Acids Res. 2019;47(D1):D411-D8.

412 7. Pellegrini M, Marcotte EM, Thompson MJ, Eisenberg D, Yeates TO. Assigning protein functions

413 by comparative genome analysis: protein phylogenetic profiles. Proc Natl Acad Sci U S A.

414 1999;96(8):4285-8.

415 8. Weissenborn S, Walther D. Metabolic Pathway Assignment of Plant Genes based on Phylogenetic Profiling-A Feasibility Study. Front Plant Sci. 2017;8:1831. 9. Sun J, Li Y, Zhao Z. Phylogenetic profiles for the prediction of protein-protein interactions: how to select reference organisms? Biochem Biophys Res Commun. 2007;353(4):985-91.

10. Ranea JA, Yeats C, Grant A, Orengo CA. Predicting protein function with hierarchical phylogenetic profiles: the Gene3D Phylo-Tuner method applied to eukaryotic genomes. PLoS computational biology. 2007;3(11):e237.

11. Kim Y, Subramaniam S. Locally defined protein phylogenetic profiles reveal previously missed protein interactions and functional relationships. Proteins. 2006;62(4):1115-24.

12. Giege R, Springer M. Aminoacyl-tRNA Synthetases in the Bacterial World. EcoSal Plus. 2016;7(1).

13. Leipe DD, Wolf YI, Koonin EV, Aravind L. Classification and evolution of P-loop GTPases and related ATPases. J Mol Biol. 2002;317(1):41-72.

427 14. Burroughs AM, Aravind L. The Origin and Evolution of Release Factors: Implications for

428 Translation Termination, Ribosome Rescue, and Quality Control Pathways. Int J Mol Sci. 2019;20(8). termination intermediates trapped by the Apidaecin 137 peptide during RF3-mediated recycling of RF1. Nat Commun. 2018;9(1):3053.

432 16. Na SI, Kim YO, Yoon SH, Ha SM, Baek I, Chun J. UBCG: Up-to-date bacterial core gene set and pipeline for phylogenomic tree reconstruction. J Microbiol. 2018;56(4):280-5.

434 17. Chen $\mathrm{H}$, Song $\mathrm{R}$, Wang $\mathrm{G}$, Ding Z, Yang $\mathrm{C}$, Zhang J, et al. OLA1 regulates protein synthesis and integrated stress response by inhibiting elF2 ternary complex formation. Sci Rep. 2015;5:13241.

18. Balasingam N, Brandon HE, Ross JA, Wieden HJ, Thakor N. Cellular roles of the human Obg-like ATPase 1 (hOLA1) and its YchF homologs. Biochem Cell Biol. 2019:1-11.

438 19. Tautz D, Domazet-Loso T. The evolutionary origin of orphan genes. Nat Rev Genet. 439 2011;12(10):692-702.

440 20. Khalturin K, Hemmrich G, Fraune S, Augustin R, Bosch TC. More than just orphans: are 441 taxonomically-restricted genes important in evolution? Trends Genet. 2009;25(9):404-13.

442 21. Wilson GA, Feil EJ, Lilley AK, Field D. Large-scale comparative genomic ranking of taxonomically restricted genes (TRGs) in bacterial and archaeal genomes. PLoS One. 2007;2(3):e324. 
22. Wilson GA, Bertrand N, Patel Y, Hughes JB, Feil EJ, Field D. Orphans as taxonomically restricted and ecologically important genes. Microbiology. 2005;151(Pt 8):2499-501.

23. Graham DE, Overbeek R, Olsen GJ, Woese CR. An archaeal genomic signature. Proc Natl Acad Sci U S A. 2000;97(7):3304-8.

24. Mushegian AR, Koonin EV. A minimal gene set for cellular life derived by comparison of complete bacterial genomes. Proc Natl Acad Sci U S A. 1996;93(19):10268-73.

25. Koonin EV. Comparative genomics, minimal gene-sets and the last universal common ancestor. Nat Rev Microbiol. 2003;1(2):127-36.

26. Lagesen K, Ussery DW, Wassenaar TM. Genome update: the 1000th genome--a cautionary tale. Microbiology. 2010;156(Pt 3):603-8.

27. Charlebois RL, Doolittle WF. Computing prokaryotic gene ubiquity: rescuing the core from extinction. Genome Res. 2004;14(12):2469-77.

28. Wissler L, Gadau J, Simola DF, Helmkampf M, Bornberg-Bauer E. Mechanisms and dynamics of orphan gene emergence in insect genomes. Genome Biol Evol. 2013;5(2):439-55.

29. Prabh N, Roeseler W, Witte H, Eberhardt G, Sommer RJ, Rodelsperger C. Deep taxon sampling reveals the evolutionary dynamics of novel gene families in Pristionchus nematodes. Genome Res. 2018;28(11):1664-74.

30. Koonin EV. Evolution of the Genomic Universe. In: V. K, C. M, S. I-V, C S, editors. Genetics, Evolution and Radiation. New York: Springer International; 2016. p. 413-40.

31. Zhang J, Dean AM, Brunet F, Long M. Evolving protein functional diversity in new genes of Drosophila. Proc Natl Acad Sci U S A. 2004;101(46):16246-50.

32. Zhang J, Yang H, Long M, Li L, Dean AM. Evolution of enzymatic activities of testis-specific shortchain dehydrogenase/reductase in Drosophila. J Mol Evol. 2010;71(4):241-9.

33. Long $M$, Betran $E$, Thornton $K$, Wang $W$. The origin of new genes: glimpses from the young and old. Nat Rev Genet. 2003;4(11):865-75.

34. Long $\mathrm{M}$, Langley $\mathrm{CH}$. Natural selection and the origin of jingwei, a chimeric processed functional gene in Drosophila. Science. 1993;260(5104):91-5.

35. Wang W, Zhang J, Alvarez C, Llopart A, Long $M$. The origin of the Jingwei gene and the complex modular structure of its parental gene, yellow emperor, in Drosophila melanogaster. Mol Biol Evol. 2000;17(9):1294-301.

36. Bai Y, Casola C, Feschotte C, Betran E. Comparative genomics reveals a constant rate of origination and convergent acquisition of functional retrogenes in Drosophila. Genome Biol. 2007;8(1):R11.

37. Chen S, Ni X, Krinsky BH, Zhang YE, Vibranovski MD, White KP, et al. Reshaping of global gene expression networks and sex-biased gene expression by integration of a young gene. EMBO J. 2012;31(12):2798-809.

38. Chen S, Krinsky BH, Long M. New genes as drivers of phenotypic evolution. Nat Rev Genet. 2013;14(9):645-60.

39. Vinogradov SN, Hoogewijs D, Bailly X, Arredondo-Peter R, Gough J, Dewilde S, et al. A phylogenomic profile of globins. Bmc Evol Biol. 2006;6:31.

40. Tan CL. Holistic Study of Whole Genomes. J Genome. 2017;1(1):1000e102.

41. Toll-Riera M, Bosch N, Bellora N, Castelo R, Armengol L, Estivill X, et al. Origin of primate orphan genes: a comparative genomics approach. Mol Biol Evol. 2009;26(3):603-12.

42. Palmieri N, Kosiol C, Schlotterer C. The life cycle of Drosophila orphan genes. Elife. 2014;3:e01311.

43. Arendsee ZW, Li L, Wurtele ES. Coming of age: orphan genes in plants. Trends Plant Sci. 2014;19(11):698-708. 
44. Bowling BV, Schultheis PJ, Strome ED. Implementation and assessment of a yeast orphan gene research project: involving undergraduates in authentic research experiences and progressing our understanding of uncharacterized open reading frames. Yeast. 2016;33(2):43-53.

45. Bianchi MM, Sartori G, Vandenbol M, Kaniak A, Uccelletti D, Mazzoni C, et al. How to bring orphan genes into functional families. Yeast. 1999;15(6):513-26.

46. Prabh N, Rodelsperger $\mathrm{C}$. Are orphan genes protein-coding, prediction artifacts, or non-coding RNAs? BMC Bioinformatics. 2016;17(1):226.

47. Prabh N, Rodelsperger C. De Novo, Divergence, and Mixed Origin Contribute to the Emergence of Orphan Genes in Pristionchus Nematodes. G3 (Bethesda). 2019;9(7):2277-86.

48. McLysaght A, Hurst LD. Open questions in the study of de novo genes: what, how and why. Nat Rev Genet. 2016;17(9):567-78.

49. Schmitz JF, Bornberg-Bauer E. Fact or fiction: updates on how protein-coding genes might emerge de novo from previously non-coding DNA. F1000Res. 2017;6:57.

50. Wu B, Knudson A. Tracing the De Novo Origin of Protein-Coding Genes in Yeast. MBio. 2018;9(4).

51. Van Oss SB, Carvunis AR. De novo gene birth. PLoS Genet. 2019;15(5):e1008160.

52. Zhang L, Ren Y, Yang T, Li G, Chen J, Gschwend AR, et al. Rapid evolution of protein diversity by de novo origination in Oryza. Nat Ecol Evol. 2019;3(4):679-90.

53. Johnson BR, Tsutsui ND. Taxonomically restricted genes are associated with the evolution of sociality in the honey bee. BMC Genomics. 2011;12:164.

54. Tan CL. Using Taxonomically Restricted Essential Genes to Determine Whether Two Organisms Can Belong to the Same Family Tree. Answers Research Journal. 2015;8:413-35.

55. Verster AJ, Styles EB, Mateo A, Derry WB, Andrews BJ, Fraser AG. Taxonomically Restricted Genes with Essential Functions Frequently Play Roles in Chromosome Segregation in Caenorhabditis elegans and Saccharomyces cerevisiae. G3 (Bethesda). 2017;7(10):3337-47.

56. Johnson BR. Taxonomically Restricted Genes Are Fundamental to Biology and Evolution. Front Genet. 2018;9:407.

57. Hanson AD, Pribat A, Waller JC, de Crécy-Lagard V. 'Unknown' proteins and 'orphan' enzymes: the missing half of the engineering parts list - and how to find it. Biochem J. 2009;425:1-11. 


\section{Additional information}

523 S1 Fig. Change of numbers of genes in different gene groups in 12 additional species

524 Note that the first vertical line in each panel is not its $\mathrm{Y}$-axis but the orphan genes in the corresponding

525 organism. The numbers in the parenthesis next to the name of an organism is the number of its orphan

526 gene, the total protein-coding gene in its genome, and the percent of the orphan genes of the total.

527 S2 Table. Number of genes in different gene groups for all NMS

528 S3 Table. Distribution of orphan, nearly-orphan, universal, and nearly-universal genes of

529 all NMS. The 17 chosen species were highlighted with red font.

530 S4 Table. Weighted distribution of orphan, nearly-orphan, universal, and nearly-universal

531 genes of all NMS

532 S5 Table. Analysis of universal genes of selected organisms. Reference genes are

533 highlighted with red font. The seemingly out-of-place homologs are shaded with yellow.

534 S6 Table. Analysis of universal genes of unknown identity. Reference genes are

535 highlighted with red font.

536 S7 Table. Analysis of the identity of universal genes of all NMS 
A. Escherichia coli (332/4306, 7.7\%)

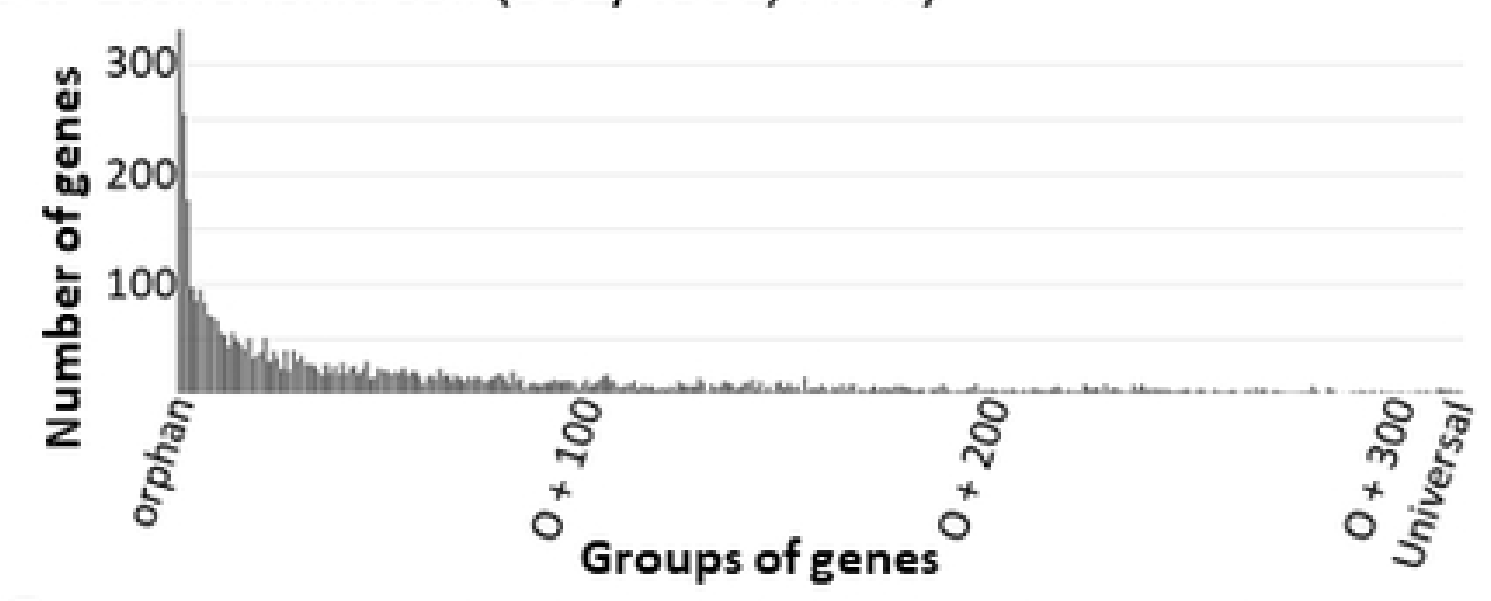

C. Drosophila melanogaster (3189/13704, 23.3\%)

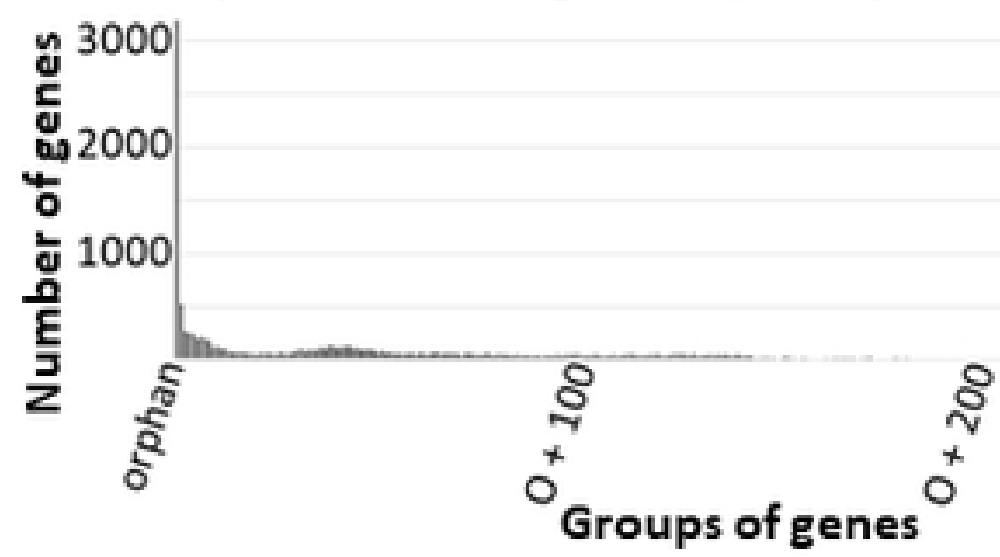

E. Homo sapiens (896/21006, 4.3\%)

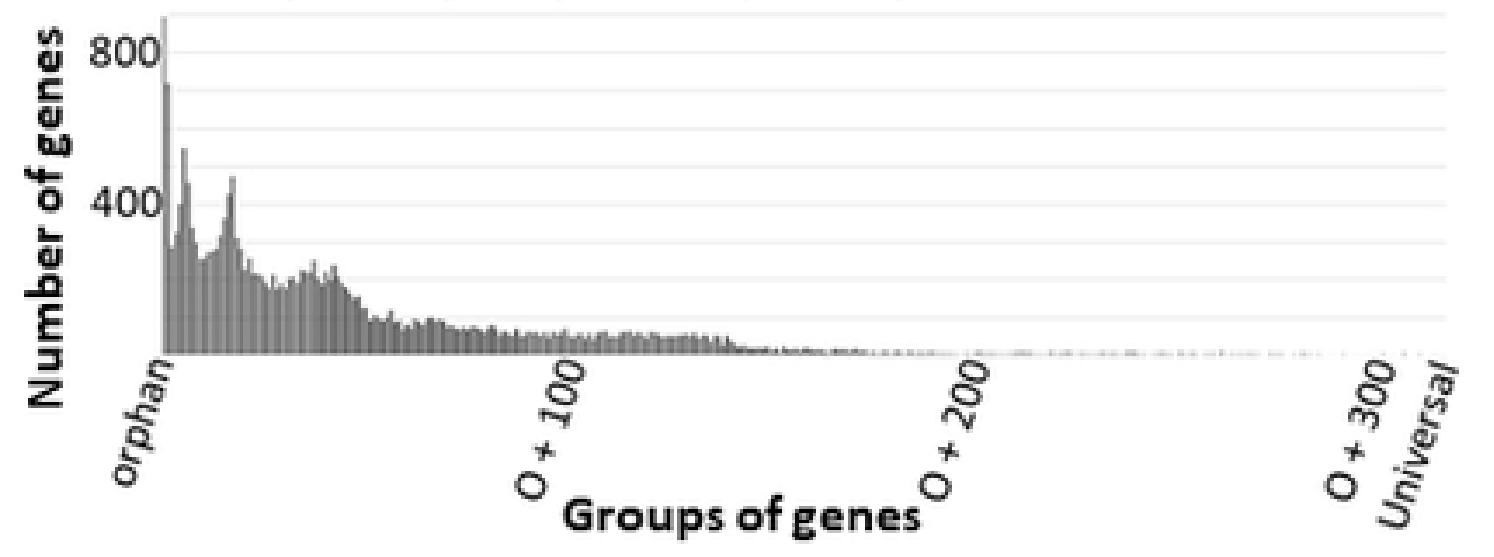

B. Saccharomyces cerevisiae (1955/6721, 29.1\%)

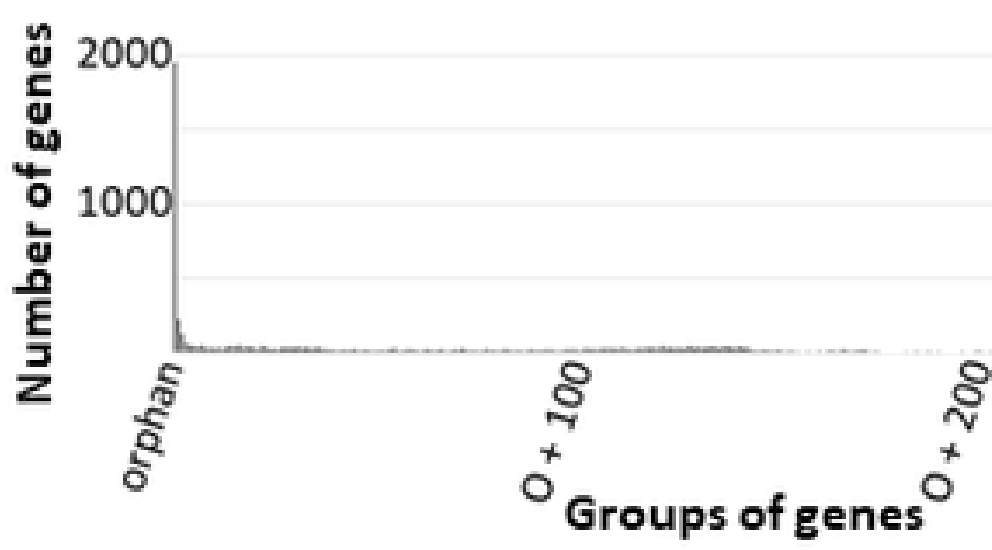

D. Mus musculus (1414/22274, $6.3 \%)$
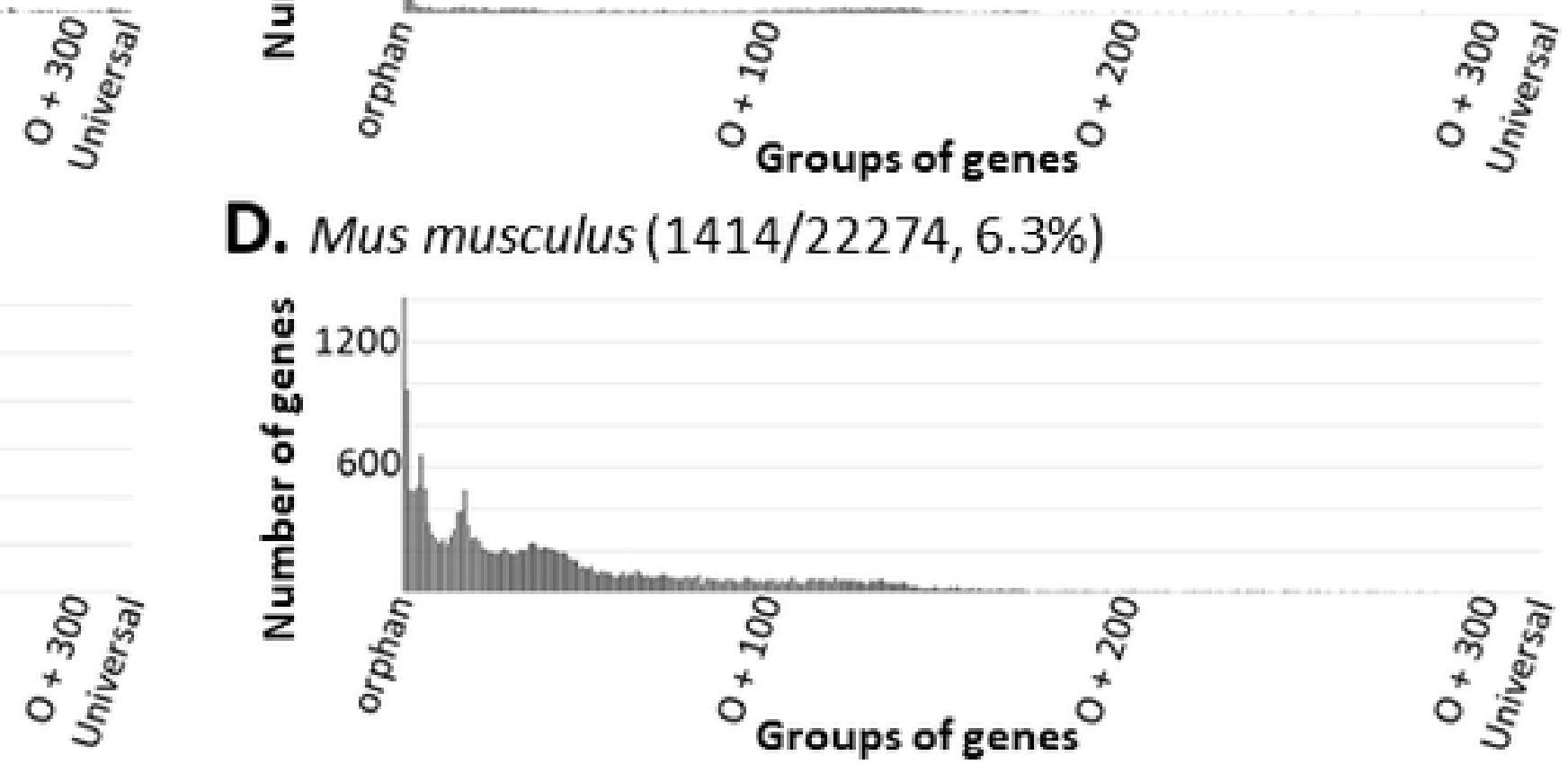

๖े

Figure 2 


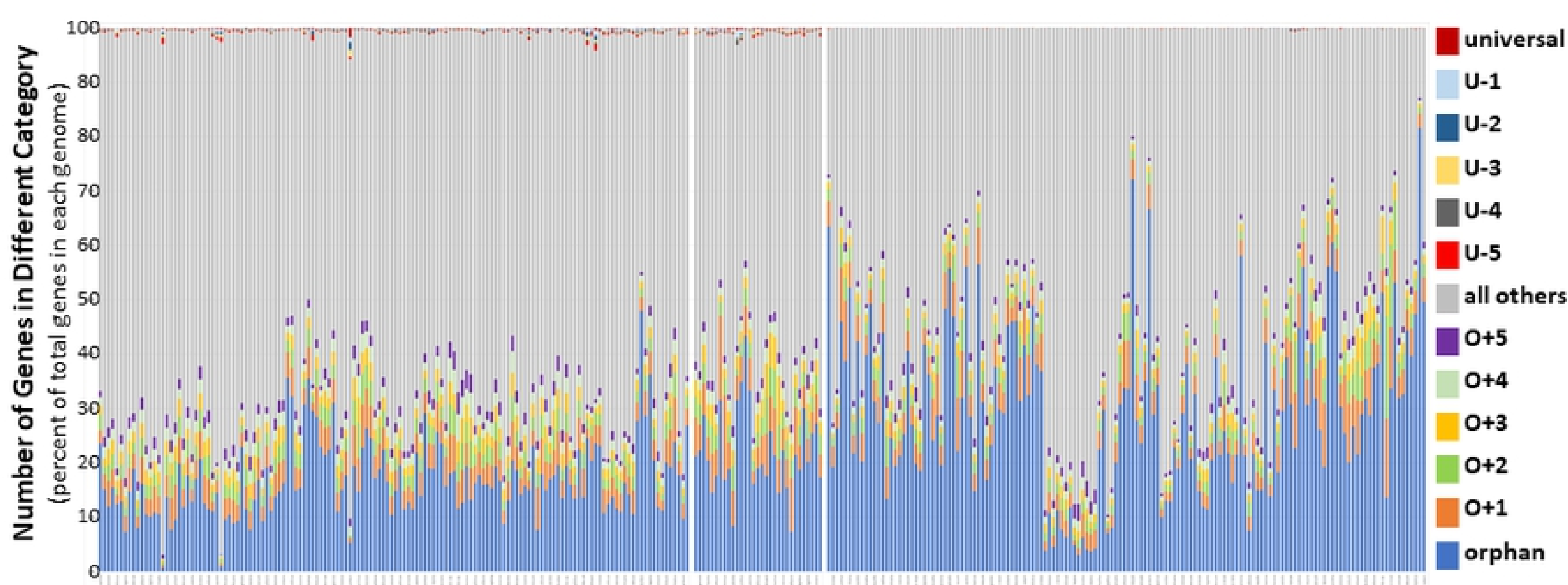




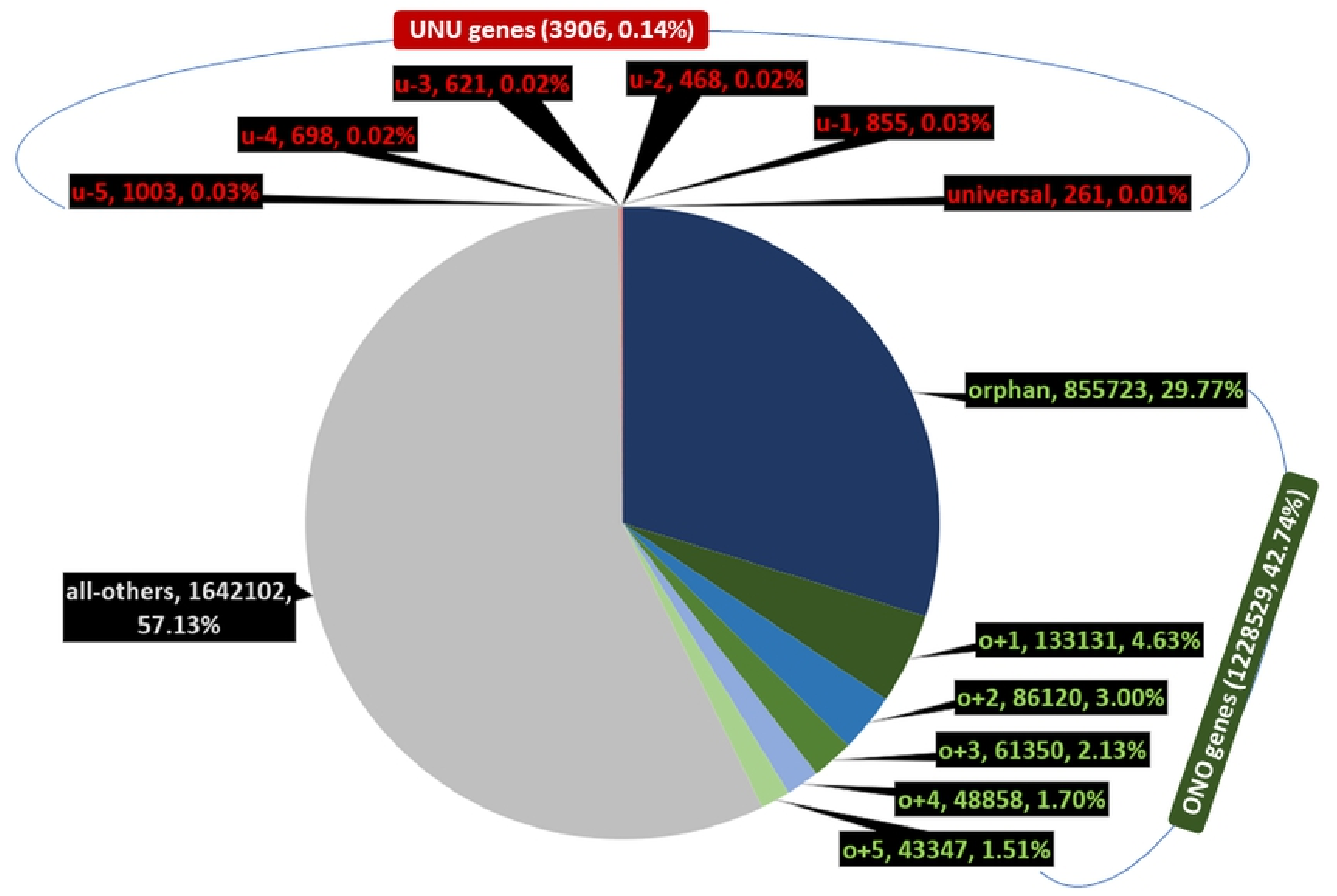

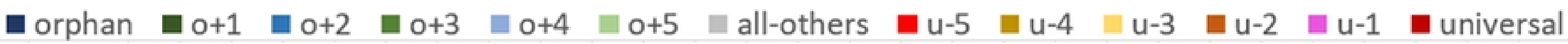
Figure 4 


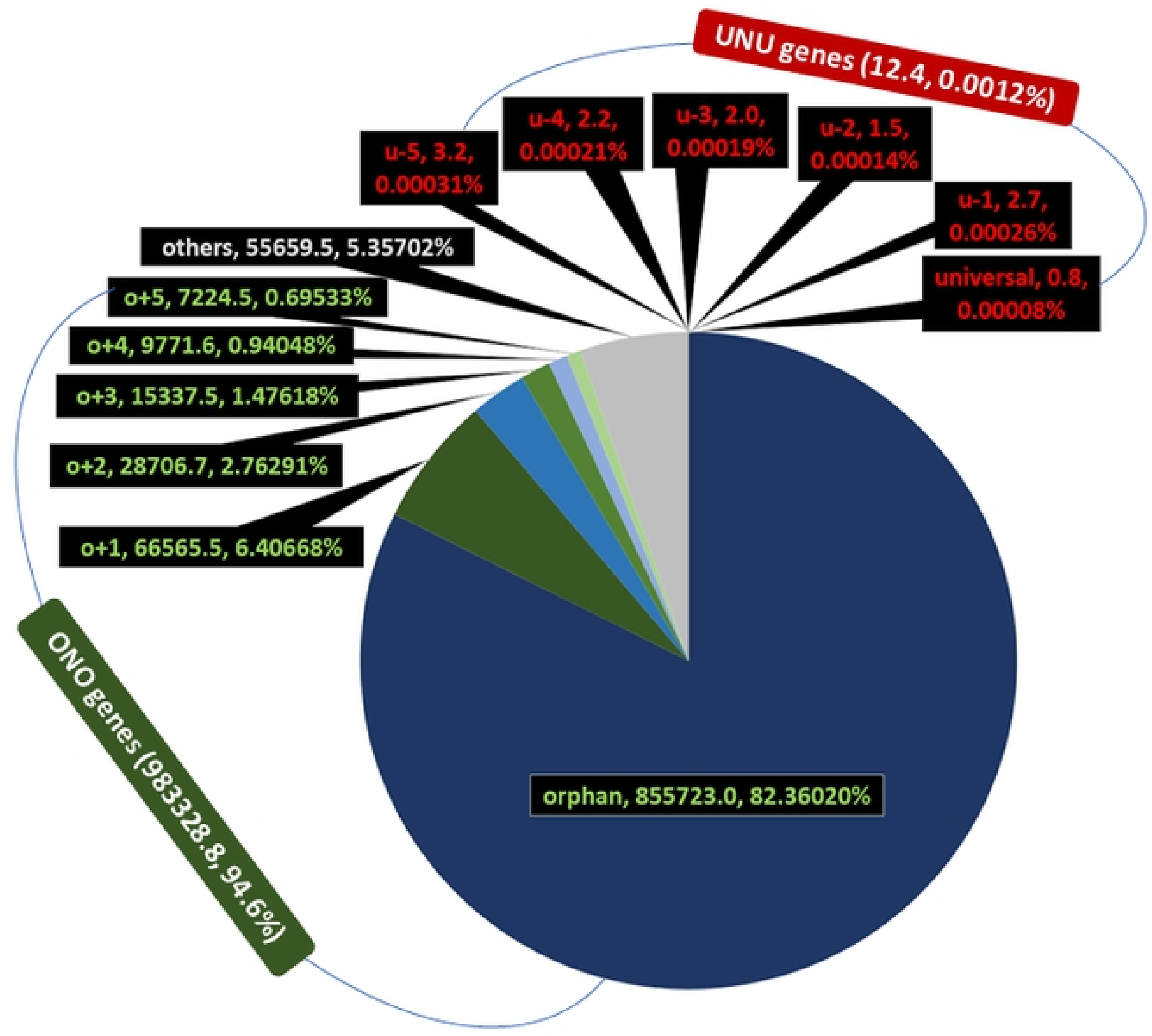

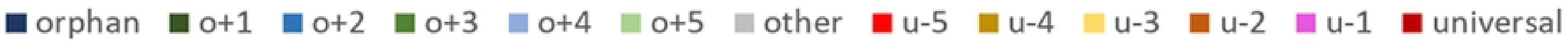
Figure 5 

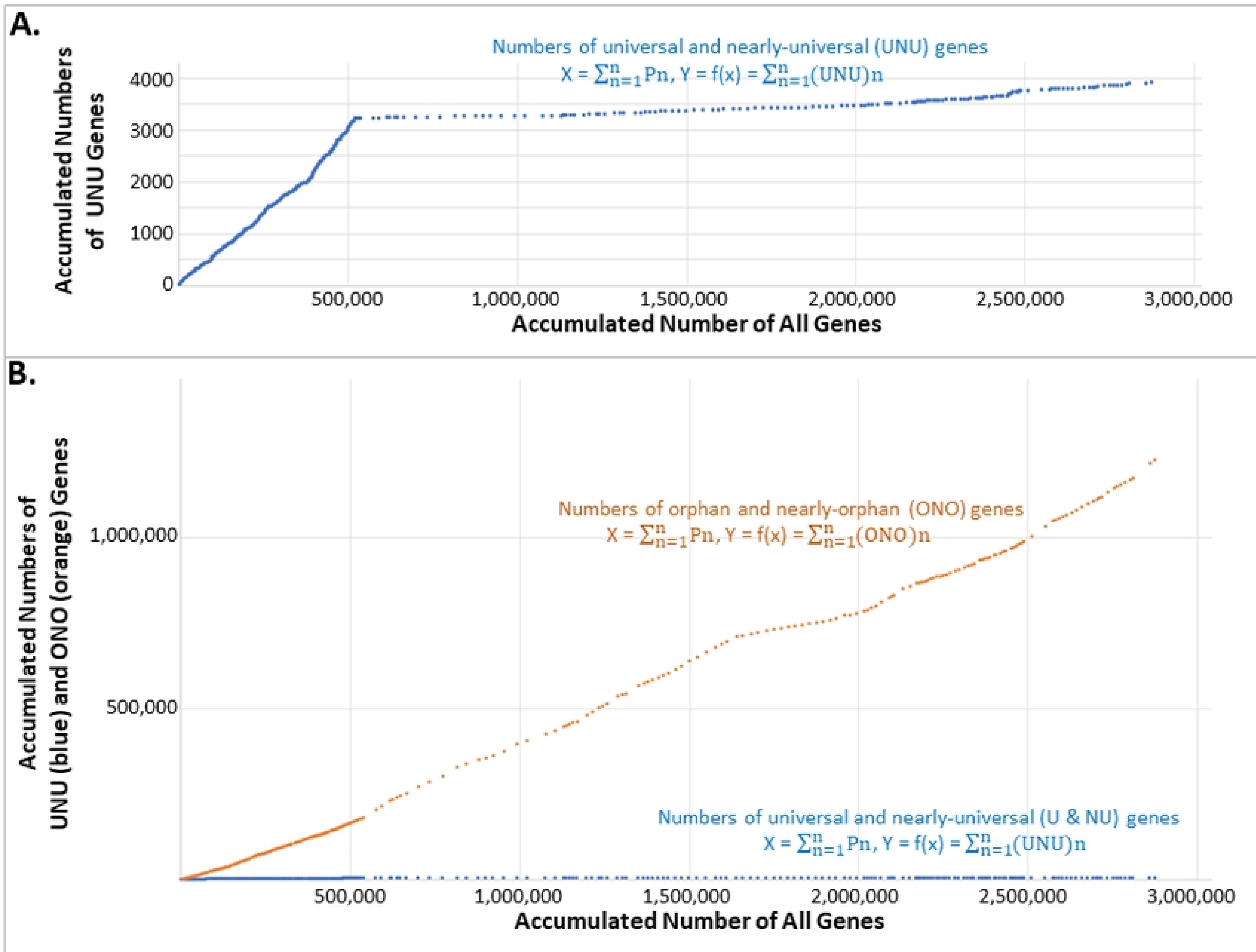

Figure 6 

RpbA'

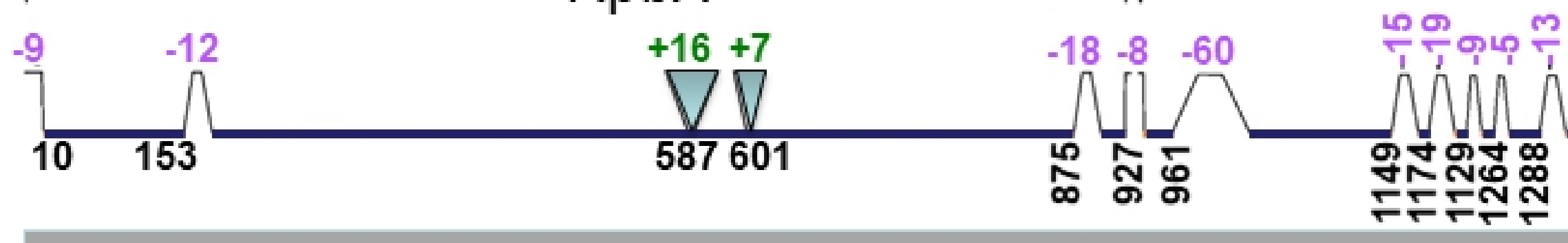

Figure 8 